\title{
Assess Nurses’ Knowledge and Attitude for Patient Safety in Cardiac Catheterization Unit
}

Shimaa Mohammed Hasballah ${ }^{1}$, Olfat Abd Elgany Shaor ${ }^{2}$, Mona Aly Mohamed ${ }^{3}$ \& Ayman Khairy Mohamed ${ }^{4}$.

1. Professional nurse at Heart Hospital, Assiut University Egypt,

2. Assistant Professor of Critical Care Nursing Department, Faculty of Nursing, Assuit University Egypt,

3. Assistant Professor of Critical Care Nursing Department, Faculty of Nursing, Assuit University Egypt,

4. Assistant Professor of Cardiology Department, Faculty of Medicine, Assuit University Egypt

\begin{abstract}
Background: Patient safety is a major component of healthcare quality, which is defined as the avoidance of causing harm to patients in providing healthcare services. The cardiac catheterization is a procedure that allows a cardiologist to get firsthand information about the patterns of blood pressure and blood flow inside the heart. Aim of the study To assess nurses knowledge and attitude for patient safety in cardiac catheterization unit. A Descriptive research design was adopted to conduct this study. The study was conducted in cardiac catheterization unit at Heart Hospital, Assuit university. Subjects The study included 40 nurses working in mentioned setting. Tools: A structured interview sheet was conducted, structured into three parts included personal data, nurses' knowledge and nurses' attitude for patient safety. Results: It was found that all of nurses (100\%) had poor knowledge for patient safety. While $(77.5 \%)$ of nurses had negative attitude and $(22.5 \%)$ of them had positive attitude toward patient safety in cardiac catheterization unit. Conclusion: it was concluded that nurses in cardiac catheterization unit had poor knowledge and negative attitude for patient safety. Recommendations: Educational program should be done for nurses in cardiac catheterization about patient safety to improve their knowledge and attitude for patient safety.
\end{abstract}

\section{Keywords: Cardiac Catheterization, Nurses' Knowledge \& Nurses' Attitude.}

\section{Introduction}

Coronary Artery Disease (CAD) is the main causes of death in both men and women. Cardiac catheterization is considered the effective diagnosing, evaluating, and treating method of Cardiac diseases problems. Although it has decreasing mortality and morbidity for cardiovascular disease, this procedure has many of complications (Younus, 2018).

Cardiac catheterization is an invasive procedure which is used in the diagnosis and treatment of several cardiac diseases. It may lead to several major and minor complications which may contribute to morbidity and mortality. Early recognition of complications and proper care is logically tied to taking action to receive prompt treatment and thus minimizing further complications (Wankhede \& Biradar, 2019).

Tests and invasive diagnostic interference including right and left cardiac catheterization have become routine diagnostic material in cardiac departments around worldwide. The method includes admission of sheaths where femoral and radial arteries are the location for admission insertion. However, femoral sheath is the most common location of admission for the sheath (Hassan, 2017).

However, as the number of procedures undertaken in the cardiac catheterization laboratories (CCL) increases, so does the potential for error and patient harm. Complex environments such as CCLs and operating theatres are high-risk areas for patient safety incidents (PSIs), and complications during and after percutaneous procedures continue to represent a significant cause of morbidity and mortality (Lindsay, et al., 2018).

Patient safety is a serious global public health issue. Occasionally when people receive health care, errors associated with care may result in a serious harm such as death, disability or additional prolonged treatment. In addition, it may also cause indirect health care costs and productivity losses; it also affects the customer perception, attitude and trust. On the other hand, it may also affect providers 'confidence and integrity (El-Hosany, et al., 2019).

Patient safety is defined as being free from accidental harm as a result of a health care encounter. It is the responsibility of the cardiac catheterization team to make that commitment to every patient and to each other. A well-functioning unit with a culture of safety, demonstrated clinical quality outcomes, and high internal/external customer satisfaction scores can avoid the risks associated with a less reliable unit (Keshk \& Elgazzar, 2108).

Assessment of the attitude of nurses toward the patient safety culture in hospitals reveals the dimensions that need to be improved, thereby allowing the implementation of proper interventions to endorse the patient safety culture (Saberi, et al., 2017). Nurses in the Cardiac Catheterization Lab (CCL) play a vital part in providing quality care to their patients. 
Through knowledge and current evidence based practice, is the key to become an effective and efficient nurse. CC staff and nurses whose caring for post cardiac catheterization procedure patients should be work together to reduce complications, when possible, and treat these complications when they occur (Feroze, et al., 2017).

\section{Significance of the study}

Few medical settings have seen such rapid growth in activity over the last two decades as the cardiac catheterization laboratory (CCL) (Lindsay, et al., 2018).

In Assiut University Heart Hospital, according to patient record in cardiac catheterization unite, number of patient admission during 2016 were (4 thousand) per year including (cardiac angiography, percutaneous coronary intervention, implantation of pacemaker). Therefore the study will be conducted to assess knowledge and attitude of nurses about patient safety in cardiac catheterization unit (Cardiac Catheterization unit, Assiut University hospital Egypt, 2016).

\section{Aim of the study}

To assess nurses knowledge and attitude for patient safety in cardiac catheterization unit.

Research questions:

1. What is the knowledge of nurses for patient safety in cardiac catheterization unit?

2. What is the attitude of nurses for patient safety in cardiac catheterization unit?

\section{Subjects \& Method}

Study desig:

- A Descriptive research design was utilized to conduct the aim of this study.

Setting

- The study was conducted in cardiac catheterization unit at Heart Hospital, Assiut University. It consists of three operating rooms, two recovery rooms and patient preparation room.

\section{Study Subjects}

- All available nurses were selected in the study; the sample of this study was consisted of 40 nurses.

\section{Study tools}

Tool I: A structured interview questionnaire was conducted after reviewing the relevant literature to elicit information. It included three parts.

\section{The first part}

It included nurses demographic data such as age, sex, marital status, level of education, and experience years in cardiac catheterization unit.

\section{The second Part}

It included (34 items) multiple choice questions regarding nurses' knowledge about cardiac catheterization (5 items), patient safety (10 items), and most common adverse event (4 items), contributing factors (4 items), patient safety culture (7 items), and complications related to cardiac catheterization (4 items).

Scoring system for knowledge: The total score of knowledge were 34 grads it developed as one grad for each correct answer and zero for each incorrect answer. For each area of knowledge, the scores of the items were summed-up and the total divided by the number of the items, giving a mean score for the part. These scores were converted into a percent score as the following. Poor $<75 \%$, Good $>75 \%$.

\section{Third Part}

Nurses attitude toward patient safety in cardiac catheterization unit. It consists of (8) items that show nurses opinion about patient safety in cardiac catheterization unit. Their opinion measured by 5point Likert scale (1=disagree strongly, 2-disagree slightly, 3=neutral, 4=agree slightly, 5= agree strongly). This part was developed by the researcher based on safety attitude questionnaire (SAQ) which was developed by (Saberi, et al., 2017).

Scoring system for attitude: The total score of attitude were 40 grades; it developed as (1=disagree strongly, 2=disagree slightly, 3=neutral, 4=agree slightly, 5= agree strongly). For each area of attitude, the scores of the items were summed-up and the total divided by the number of the items, giving a mean score for the part. These scores were converted into a percent score as the following. Negative attitude $<50 \%$, positive attitude $>50 \%$.

\section{Method}

\section{Preparatory phase}

- An official permission from the dean of faculty of nursing to conduct this study was delivered to the head of the Heart Hospital at Assiut University and approval to conduct this study was obtained after explanation of the aims of study.

- Permission for voluntary participation was obtained from nurses and the nature and purpose of the study was explained by the researcher.

\section{Content validity}

The developed tools were tested for content related validity by a jury of (7) experts in the field of the study and the necessary modifications were done. 3 from coronary care medicine Assiut University faculty of medicine and 4 from critical care nursing in assiut faculty of nursing. The overall reliability of the tools was tested using alpha Cronbach's values 0.84 of the tools. 


\section{Pilot Study}

After developing the necessary tool of the study. A Pilot study included $10 \%$ of nurses was carried out to ensure clarity and applicability of the developed tool, and to estimate the time required to fill the questionnaire. Based on the results of the pilot study, the necessary modifications were done nurses included in the pilot study were excluded from the study sample.

\section{Ethical consideration}

Research proposal will be approved from Ethical committee in the Faculty of Nursing at Assiut University. There is no risk for study subject during application of the research. The study will follow common ethical principles in clinical research. Confidentiality and anonymity will assured. Study subject have the right to refuse to participate and or withdraw from the study without rational any time. Study subject privacy will be considered during collection of data.

Agree to participant in this study (oral agreement).

\section{2-Impelmentation phase}

- An interview form was designed by researcher who visited the selected setting and requested from nurses to participate in the study through face-to-face interviews

- The questionnaire was adopted by the researcher in Arabic format in order to suit the studied nurses' language and their level understanding.

- The researcher had made an interview and asked nurses individually to fill out structured questionnaire at break time to identify nurses' knowledge and attitude about patient safety in cardiac catheterization.

- The researcher had met one or two nurses each time to fill out the questionnaire at morning shift and each interview had taken half hour.

- The interview was conducted in cardiac catheterization unite at Assiut University heart hospital.

- -The nurses were assessed their knowledge about patient safety in cardiac catheterization unite (Second part).

- The nurses were assessed their attitude about patient safety in cardiac catheterization unite (Third part).

\section{Statistical analysis}

Data entry and data analysis were done using SPSS version 19 (Statistical Package for Social Science). Data were presented as number, percentage, mean+standard deviation. Chi-square test and Fisher Exact test were used to compare qualitative variables. Pvalue considered statistically significant when $\mathrm{P}<$ 0.05 . 


\section{Results}

Table (1): Distribution of nurses regarding their socio demographic characteristics in cardiac catheterization unit (n=40).

\begin{tabular}{|c|c|c|}
\hline Variables & $(n=40)$ & $\%$ \\
\hline \multicolumn{3}{|l|}{ Sex } \\
\hline Male & 15 & 37.5 \\
\hline Female & 25 & 62.5 \\
\hline \multicolumn{3}{|l|}{ Age group } \\
\hline$<25$ years & 15 & 37.5 \\
\hline $25-<30$ years & 11 & 27.5 \\
\hline$>30$ years & 14 & 35.0 \\
\hline Mean \pm SD(range) & \multicolumn{2}{|c|}{$29.25 \pm 7.07(21-49)$} \\
\hline \multicolumn{3}{|l|}{ Marital Status } \\
\hline Single & 16 & 40.0 \\
\hline Married & 24 & 60.0 \\
\hline \multicolumn{3}{|l|}{ Education Level } \\
\hline Nursing Diploma & 15 & 37.5 \\
\hline nursing Institute & 15 & 37.5 \\
\hline Bachelor of Nursing & 10 & 25.0 \\
\hline \multicolumn{3}{|c|}{ Experience years in cardiac catheterization } \\
\hline Less than 5 years & 23 & 57.5 \\
\hline from 5- 10 years & 3 & 7.5 \\
\hline More than 10 years & 14 & 35.0 \\
\hline Mean \pm SD(range) & \multicolumn{2}{|c|}{$7.39 \pm 7.17(1-22)$} \\
\hline \multicolumn{3}{|l|}{ Training courses } \\
\hline Yes & 0 & 00 \\
\hline No & 40 & 100 \\
\hline
\end{tabular}

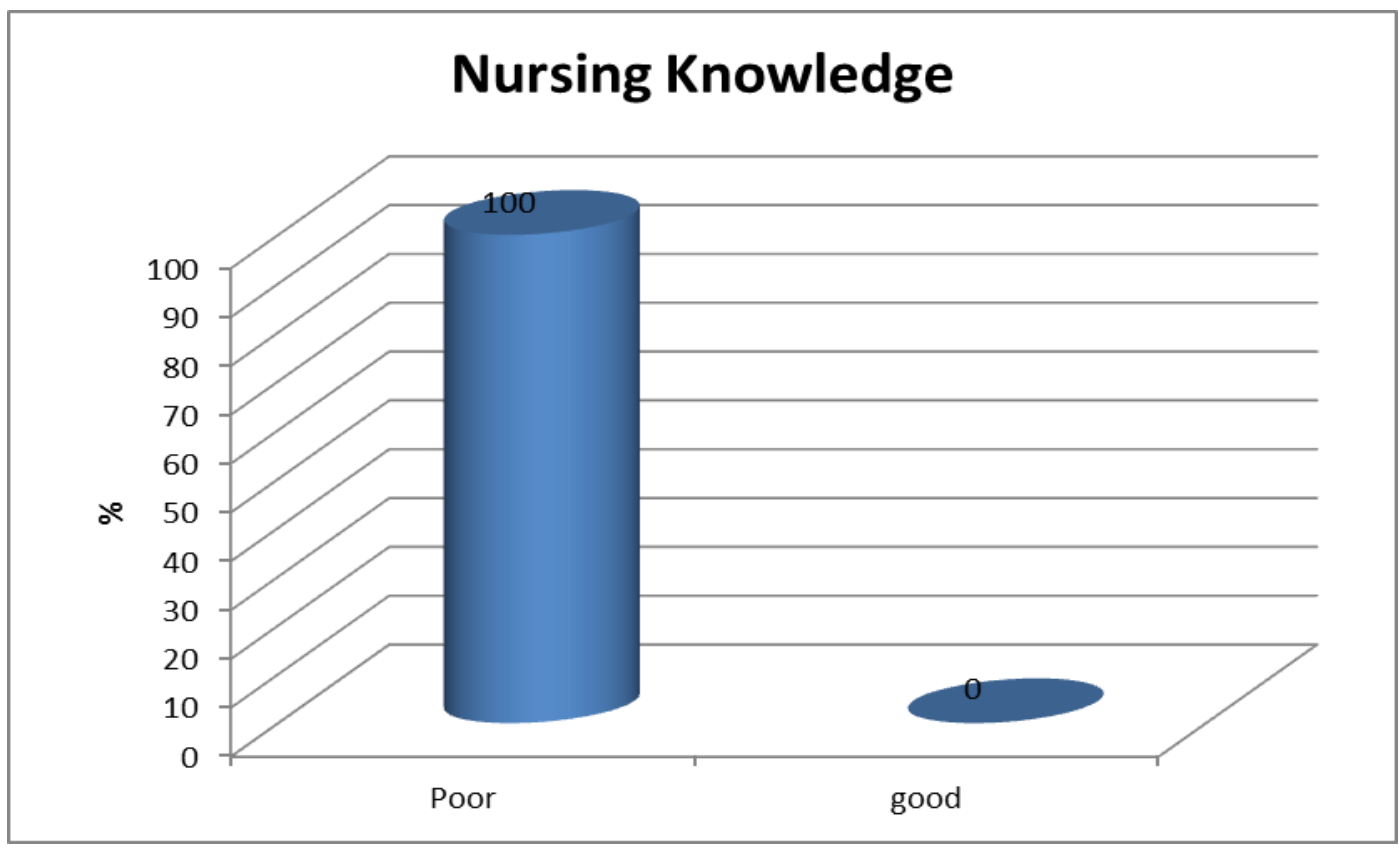

Figure (1): Distribution of nurses' knowledge score regarding patient safety in cardiac catheterization unit. 
Table (2): Relationship between nurses' knowledge score about patient safety with their Socio demographic data in cardiac catheterization unit $(n=40)$.

\begin{tabular}{|c|c|c|c|c|}
\hline \multirow{2}{*}{ Variables } & \multirow{2}{*}{$\mathbf{n}$} & \multicolumn{3}{|c|}{ Score } \\
\hline & & Mean \pm SD & Range & P. value \\
\hline \multicolumn{5}{|l|}{ Gender } \\
\hline Male & 15 & $18.3 \pm 2.86$ & $15-24$ & \multirow{2}{*}{0.142} \\
\hline Female & 25 & $16.76 \pm 3.19$ & $13-24$ & \\
\hline \multicolumn{5}{|l|}{ Age group } \\
\hline$<25$ years & 15 & $16.46 \pm 1.96$ & $13-20$ & \multirow{3}{*}{0.047} \\
\hline $25-<30$ years & 11 & $19.27 \pm 3.72$ & $13-24$ & \\
\hline$>30$ years & 14 & $16.7 \pm 3.17$ & $14-23$ & \\
\hline \multicolumn{5}{|l|}{ Marital Status } \\
\hline Single & 16 & $17.63 \pm 2.09$ & $16-23$ & \multirow{2}{*}{0.626} \\
\hline Married & 24 & $17.13 \pm 3.69$ & $13-24$ & \\
\hline \multicolumn{5}{|l|}{ Education Level } \\
\hline Nursing Diploma & 15 & $15.33 \pm 1.92$ & $13-21$ & \multirow{3}{*}{0.002} \\
\hline nursing Institute & 15 & $17.93 \pm 2.63$ & $15-23$ & \\
\hline Bachelor of Nursing & 10 & $19.4 \pm 3.72$ & $13-24$ & \\
\hline \multicolumn{5}{|l|}{$\begin{array}{l}\text { Experience year in cardiac } \\
\text { catheterization }\end{array}$} \\
\hline Less than 5 years & 23 & $17.83 \pm 3.1$ & $13-24$ & \multirow{3}{*}{0.501} \\
\hline from $5-10$ years & 3 & $16.33 \pm 3.51$ & $13-20$ & \\
\hline More than 10 years & 14 & $16.71 \pm 3.17$ & $14-23$ & \\
\hline
\end{tabular}

-Independent $T$ - test $*$ significant difference at $(p<0.05) \quad$ - Anova - test $*$ significant difference at $(p<0.05)$

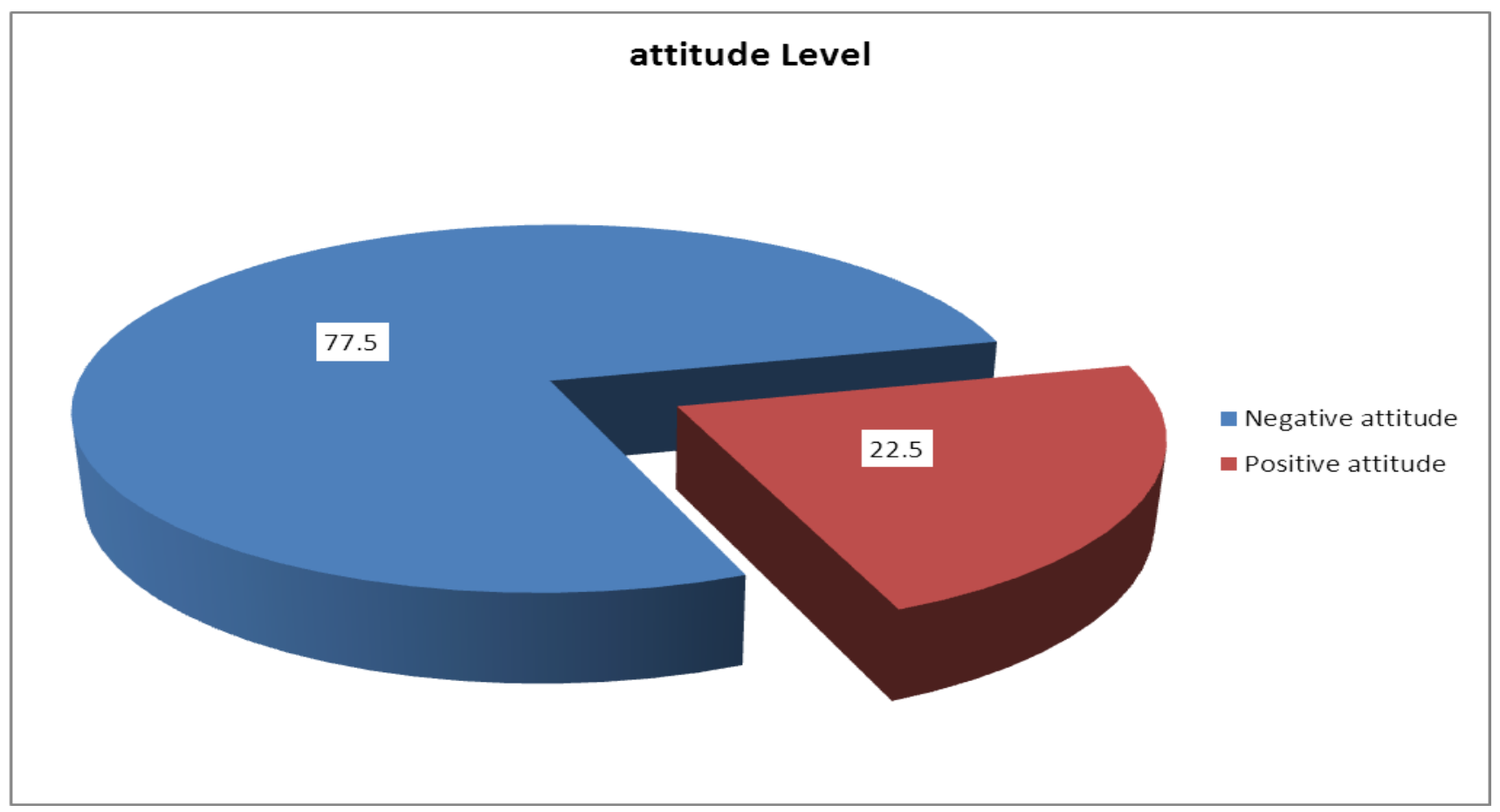

Figure (2): Distribution of nurses' attitude level toward patient safety in cardiac catheterization unit. 
Table (3): Relationship between nurses attitude toward patient safety with their Socio demographic data in cardiac catheterization unit $(n=40)$.

\begin{tabular}{|c|c|c|c|c|c|}
\hline \multirow{3}{*}{ Variables } & \multicolumn{5}{|c|}{ Nurses attitude toward patient safety } \\
\hline & \multicolumn{2}{|c|}{ Negative attitude $(n=31)$} & \multicolumn{2}{|c|}{ Positive attitude $(\mathrm{n}=9)$} & \multirow{2}{*}{ P. value } \\
\hline & No. & $\%$ & No. & $\%$ & \\
\hline \multicolumn{6}{|l|}{ Age group } \\
\hline$<25$ years & 15 & 48.4 & 0 & 0.0 & \multirow{3}{*}{0.020} \\
\hline $25-<30$ years & 8 & 25.8 & 3 & 33.3 & \\
\hline$>30$ years & 8 & 25.8 & 6 & 66.7 & \\
\hline \multicolumn{6}{|l|}{ Marital Status } \\
\hline Single & 14 & 45.2 & 2 & 22.2 & \multirow{2}{*}{0.216} \\
\hline Married & 17 & 54.8 & 7 & 77.8 & \\
\hline \multicolumn{6}{|l|}{ Education Level } \\
\hline Nursing Diploma & 9 & 29.0 & 6 & 66.7 & \multirow{3}{*}{0.119} \\
\hline nursing Institute & 13 & 41.9 & 2 & 22.2 & \\
\hline Bachelor of Nursing & 9 & 29.0 & 1 & 11.1 & \\
\hline \multicolumn{6}{|c|}{$\begin{array}{l}\text { Experience year in cardiac } \\
\text { catheterization }\end{array}$} \\
\hline Less than 5 years & 21 & 67.7 & 2 & 22.2 & \multirow{3}{*}{0.049} \\
\hline from $5-10$ years & 2 & 6.5 & 1 & 11.1 & \\
\hline More than 10 years & 8 & 25.8 & 6 & 66.7 & \\
\hline
\end{tabular}

Chi-Square Tests Statistically Significant deference at P. value $<0.05$.

Table (1): Illustrates the distribution of the nurses regarding their socio demographic characteristics, it noticed that more than half of them $(62.5 \%)$ were females. Less than half of nurses $(37.5 \%)$ were their age younger than 25 years with Mean \pm SD $29.25 \pm 7.07$ (21-49). More than half $(60 \%)$ of them were married and $(37.5 \%)$ of them have nursing diploma and equal to nursing institute. Concerning their experiences it was found that, more than half of nurses' have $(57.5 \%)$ less than five years experience with Mean \pm SD 7.39 $\pm 7.17(1-22)$. All of nurses hadn't had in-service training courses related to patient safety.

Figure (1): Show the distribution of nurses knowledge score for patient safety. It was found that all of nurses (100\%) had poor knowledge for patient safety.

Table (2): Explain the relationship between nurses' knowledge score for patient safety with their Socio demographic data. Statistical significant differences were found between knowledge scores, age groups of nurses (0.047) and educational levels among nurses $(0.002)$.

Figure (2): Explains distribution for attitude level of nurses about patient safety. It was found that (77.5\%) of nurses had negative attitude toward patient safety and $(22.5 \%)$ of them had positive attitude toward patient safety with Mean \pm SD $45.93 \pm 7.43$ and range (34 - 66).
Table (3): Explains relationship between nurses attitude toward patient safety with their demographic data by using Chi-Square Tests. Statistical significant difference was found between age groups and nurses' attitude toward patient safety (0.020). Also, statistical significant difference was found between nurses experience years in cardiac catheterization unite and nurses' attitude toward patient safety (0.049).

\section{Discussion}

The importance of patient safety is growing worldwide; and improving safe care of patients has recently evoked national attention (Findlay, Best, \& Ottrey 2016). Cardiac catheterization is a common interventional process useful for diagnosis the patients suffering from acute coronary syndrome (ACS). It can source of various health problems that have to be recognized and treated following the test. Taking into consideration probable dangerous complications so that the nurse plays a critical role in cardiac catheterization unit in the provision great quality nursing care and increase patient safety to their patients. Creature knowledgeable and existing on evidence based practice is the crucial to being an efficient and effective nurse. So nurses should understand clearly information and optimal performance about patient safety to improve quality care. (Keshk \& Elgazzar, 2018) 
The current study aimed to assess nurses' knowledge, attitude about patient safety that include nurses knowledge about cardiac catheterization, patient safety, and most common adverse event, contributing factors, patient safety culture, and complications related to cardiac catheterization.

It was observed in the present study that, more than half of them females. This agrees with (Hassan, 2017) who presented in his study about assessment of nurses knowledge about patient safety after cardiac catheterization for adult patients in Ibn Al-Biter Specialist Center Cardiac Surgery, Baghdad University that the most of nurses were female, and also agree with (Younus, 2018) that mentioned most of nurses were female.

As regarding the nurses age, the finding of the current study revealed that less than half of nurses their age was younger than 25 years. This means that most of the nurses are juniors and the advantages of nurses in young age are full of energy and hyperactivity which is always required in such crucial department. This result is supported by (Younus, 2018), (Hassan, 2017), who reported in his study, that the majority of nurses ages were ranged from 20 to 25 years old that were accounted about one third of the sample.

Regarding to the marital state, the current study clarifies that more than half of the studied nurses were married as compared to single nurses. The study results also is confirmed with (Sharif, et al., 2018) who showed that the highest percentage of the studied nurses was married. But (Feroze, et al., 2017) disagree with the current result as they found that most of nurses were unmarried.

Regarding to level of education, the current study showed that more than third of them have nursing diploma and equal to nursing institute. This is because in our country the system of the hospital for distribution of collegiate nurses at the hospital level is not appropriate and there is no functional description for nurses.

These results were agree with the studies conducted by (Feroze, et al., 2017), (Keshk \& Elgazzar, 2018) (Ali \& Ali, 2019) who reported that according to their qualification, more than half of nurses had general nursing diploma and less than half of them had BSN.

Also, the current study results revealed that more than half of nurses their experiences in cardiac catheterization were less than five years. This finding indicated that most of the nurses were juniors with little experiences. These results agree with (Feroze, et al., 2017), (Rushdy, et al., 2016) \& (Hassan, 2017) who revealed that fifty eight percent of the study samples are (1-5) years were employee in cardiac catheterization unit. But, this result mismatched with (Keshk \& Elgazzar, 2018) who reported in their study that the majority of the studied nurses had experienced more than 10 years.

In current study all nurses not attended training courses about patient safety. This means that there is no educational program conducted for nurses in CC about patient safety. Also (Ali \& Ali 2019) agree with the current study result that said all of nurses were not taking in service educational program.

As regard nurses knowledge the current study showed that all of nurses had poor knowledge about patient safety. This indicating that most of nurses working in cardiac catheterization unit were in need for more in service educational programs. These results agree with the study conducted by (Younus, 2018) that show the knowledge of nurses were poor knowledge towards patient safety after diagnostic cardiac catheterization. This result is in disagreement with (Wankhede \& Biradar, 2019), (Yaqoob, et al., 2019) who presented in his study that the majority of the study participants had adequate knowledge, less than half had inadequate knowledge, whereas only $(5.7 \%)$ had excellent knowledge.

The current study was found the association between nurse's knowledge and some socio- demographic characteristics of the sample for 40 nurses and it is present in table 2, the result showed that there are significant relationship among age groups, and level of education with nurse's knowledge. This result showed that qualification has great effect on the nurses' knowledge.

Also, the result of study which conducted by (Sharif, et al., 2018) on convenience sample of 60 nurses working in general hospital in Rania city, Iraq who reported that the association between nurse's knowledge and some socio- demographic characteristics, that there are significant relationship among age, gender, years of employment and number of training course with nurse's knowledge except level of education.

This outcome disagrees with (Ali, et al., 2015), (Feroze, et al, 2017), (Hassan, 2017), (Younus, 2018) who found no significance between knowledge score of sample and their age, level of education, and years of experience in nursing profession.

The current study results revealed that more than three quarters of nurses had negative attitude and less than one quarter of them had positive attitude for patient safety. This result agrees with study conducted by (Saberi, et al., 2017) about attitude of nurses toward the patient safety culture in Tehran, Iran that attitude of the nurses in the selected hospitals was poor toward the dimensions of the patient safety culture and lower than the international standards $(60 \%)$. 
While' this outcome disagrees with (Brasaite, 2016), (Safarpour, et al., 2017) who reported that the positive attitude of students toward medical errors reporting has a valuable effect on improving patient safety.

\section{Conclusion}

Based on the results of the present study, it was concluded that: Nurses working in cardiac catheterization unit had poor knowledge regarding patient safety. Also nurses had negative attitude toward patient safety in cardiac catheterization unit.

\section{Recommendations}

Based on results of the present study the following can be recommended:

1- Educational program for cardiac catheterization unit nurses to improve knowledge about patient safety and avoid complication after cardiac catheterization patients to develop the knowledge, and attitude needed to deal with such group of patients .

2- It is strongly recommended that specific training interventions be performed to enhance the work conditions and safety climate in these hospitals and improve nurses' attitude toward patient safety.

3- Reapply this research on a larger probability sample acquired from different geographical areas in Egypt for generalization.

\section{References}

1. Al-Ftlawy D., (2014): Determination of Nurses' Knowledge Toward Care Provided to Patients with Acute Myocardial Infarction in Al-Najaf City, Kufa Journal for Nursing Sciences, Vol.(2), No.(2).

2. Ali H., Ali M., (2019): Effect of Designed Teaching Protocol Regarding Patients' Safety after Cardiac Catheterization on Nurses' Performance and Patients' Incidence of Vascular Complications, International Journal of Studies in Nursing, Vol. (4), No.(1), Pp.107120.

3. Brasaite I., Suominen T, Kaunonen M, Haatainen D, Schoonhoven L, (2016): Health Care Professionals' Knowledge and Attitudes Regarding Patient Safety and Skills for Safe Patient Care, Tampere University Press, 9:177.

4. Cardiac Catheterization unit, Assiut University hospital Egypt, (2016): statistical record, Assiut University hospital Egypt.

5. Elfeky H., Ali F., (2013): Nurses' Practices and Perception of Delirium in the Intensive Care Units of a Selected University Hospitals in
Egypt, Journal of Education and Practice, 4(19), Pp:61-70.

6. El-Hosany W., Habashy A., Abd Elwahab E., Ali H., (2019): Developing a proposed Plan for Patients' Safety Management system in Cardiac Catheterization Units at Suez Canal University Hospitals, International Scientific Conference of Faculty of Nursing, Suez Canal University, In collaboration with Edith Cowen universityAustralia, 21-22.

7. Feroze M., Afzal M., Sarwar H., Galani A., Afshan S., (2017): Assess Knowledge and Practice of Registered Nurses about Patient Safety after Cardiac Catheterization in Punjab Institute of Cardiology Hospital, Lahore. International Journal of Musculoskeletal Pain prevention, Vol.(2), No.(2), Pp.(233-238).

8. Findlay Ú., Best H., Ottrey M., (2016): Improving patient safety in radiotherapy through error reporting and analysis, Elsevier Ltd on behalf of the college of radiographers, Vol. (22), Supplement December 2016, Pp. (S3S11).

9. Hassan A., (2017): Assessment of Nurses Knowledge about Patient Safety after Cardiac Catheterization for Adult Patients in Ibn Al Biter Specialist Center Cardiac Surgery, International Journal of Science and Research (IJSR), Vol.(6), No.(5), Pp.(2763-2766).

10. Kern M,. Lim M., Sorajja P., (2016): The cardiac catheterization handbook, E-Book, Elsevier Health Sciences, 6th ed, Pp. 470-471.

11. Keshk L., Elgazzar S., (2018): Creating Learning Guideline for Nurses Caring for Patients Safety Undergoing Cardiac Catheterization, Research Journal of Education, Vol.(4), Issue.(7),Pp.101-109.

12. Lindsay A., Bishop J., Harron K., Davies S., Haxby E., (2018): Use of a safe procedure checklist in the cardiac catheterization laboratory, BMJ Open Quality, e000074.doi:10.1136/bmjoq-2017-000074

13. Rushdy, T., Youssef, W., Elfeky, H., (2016): Nurses' knowledge and performance regarding care of patients connected to intra- aortic balloon pump at Cairo university hospitals Egyptian Nursing Journal: Available: www.elmagla.egy2010@yahoo.com

14. Saberi M., Jamshidi E., Rajabi F., Seydali E., Bairami F., (2017): Attitude of Nurses toward the Patient Safety Culture: A Cross-Sectional Study of the Hospitals in Tehran, Iran, Journal of Patient Safety and Quality Improvement ,Vol.(5), No.(3), Pp.(554- 560). 
15. Safarpour H., Tofighi M., Malekyan L., Bazyar J., (2017): Patient Safety Attitudes, Skills, Knowledge and Barriers Related to Reporting Medical Errors by Nursing Students, International Journal of Clinical Medicine, Vol.(8), Pp.(1-11)

16. Sharif B., Salih S., Sailh N., Salim B., (2018): Nurses' Knowledge Regarding Cardiac Catheterization at General Hospital in Rania City, Kurdistan Journal of Applied Research (KJAR), 2nd International Conference on the Health \& Medical Sciences, Pp:183-187.

17. Wankhede, A., \& Biradar, S., (2019): A study to Assess the Knowledge and Practices among Cardiac Nurses about Patient Safety after Cardiac Catheterization, International Journal of Science and Research (IJSR), Vol.(8),No.(6),Pp.916-920.

18. Yaqoob A., Barolia R., Noor A., Nazar A., (2019): Knowledge and Practices among Nurses Regarding Patients' Care Following Cardiac Catheterization at a Tertiary Care Hospital in Karachi, Pakistan, Open Journal of Nursing, Vol.(9), No.(8), Pp:809-834.

19. Younus, F., (2018): Nurses' Knowledge Regarding Patients Safety After Diagnostic Cardiac Catheterization in Azadi Teaching Hospital in Kirkuk City, Kirkuk University Journal /Scientific Studies (KUJSS), Vol.(13), Issue(4), pp. (45-56). 